\title{
Three-Dimensional Printing in Tissue Engineering and Regenerative Medicine
}

\author{
Chong-Su Cho', James J. Yoo \\ ${ }^{1}$ Research Institute for Agriculture and Life Sciences, Seoul National University, Seoul, Korea \\ ${ }^{2}$ Wake Forest Institute for Regenerative Medicine, Wake Forest School of Medicine, Winston-Salem, NC, USA
}

Tissue engineering strategies have been attempted to help the body's ability to regenerate damaged tissues by combination of cells, growth factors and biomaterial scaffold since Langer and Vacanti [1] firstly challenged the technique although there is a continued demand on enhancing the function of tissue-engineered organs through the development of a new fabrication method.

Recently, three-dimensional (3D) printing technologies have emerged as a powerful method in the field of tissue engineering and regenerative medicine because the inherent limitations of fabrication of 3D scaffolds can be overcome with geometric accuracy at the macroscale and microscale [2] and living cells can be included in the printing process to position living cells together with desired biomaterial scaffolds [3].

Although the optimistic promise of $3 \mathrm{D}$ printing improves tissue engineering and regenerative strategies and the field is still at a relatively early stage, many challenges such as increase of resolution, printing speed, and relevant biomaterials for creation of more complex tissue/organ structures must be solved for clinical trials [4]. Also, multidisciplinary collaborations among biologists, material scientists, engineers and medical doctors should be necessary to achieve successful 3D tissue/organ printing.

In the special issue, renowned authors in $3 \mathrm{D}$ printing fields provide a concise and up-to-date overview of different issues regarding application of 3D printing in tissue engineering and regenerative medicine. This issue covers five different topics. The first one discusses 3D cell-based bio-printing technologies and their applications in soft tissue regeneration through placement of cell-laden hydrogel-based bioinks in a layer-by-layer fashion. The second one discusses the characteristics of printing biomaterials including hydrogels, synthetic polymers and composite materials as well as recent advances in soft tissue regeneration using $3 \mathrm{D}$ printing inks. The third one discusses characteristics of the $3 \mathrm{D}$ printing techniques, used biomaterials for development of $3 \mathrm{D}$ tissue/organ analogues and in vitro models. The fourth one discusses $3 \mathrm{D}$ potential practice in regenerative medicine to fabricate the transplantable hard tissues such as cartilage and bone and current technical development of 3D bio-printing for engineering hard tissues. The fifth one discusses the history and types of 3D printers, the classfication of $3 \mathrm{D}$ printers and the technology used to manufacture artificial tissues/organs.

We expect that this review will provide scientists, researchers and healthcare industrial persons in the fields of 3D printing fundamental concepts and practical tools for their successful application of reliable fabrication techniques to replace and regenerate defective tissues or injured tissues and organs.

\section{Acknowledgements}

The editors would like to express their deepest gratitude to all the invited authors for accepting the challenge of writing this review although they must be very busy.

\section{REFERENCES}

1. Langer RS, Vacanti JP. Tissue engineering: the challenges ahead. Sci Am 1999;280:86-89.

2. Sears NA, Seshadri DR, Dhavalikar PS, Cosgriff-Hernandez E. A review of three-dimensional printing in tissue engineering. Tissue Eng Part B Rev 2016;22:298-310.

3. Park JH, Jang J, Lee JS, Cho DW. Three-dimensional printing of tissue/ organ analogues containing living cells. Ann Biomed Eng 2016 Apr 14 [Epub]. http://dx.doi.org/10.1007/s10439-016-1611-9.

4. Lee VK, Dai G. Printing of three-dimensional tissue analogs for regenerative medicine. Ann Biomed Eng 2016 Apr 11 [Epub]. http://dx.doi. org/10.1007/s10439-016-1613-7. 